\title{
Image Blur Estimation Based on the Average Cone of Ratio in the Wavelet Domain
}

\author{
Ljiljana Ilić, Aleksandra Pižurica, Ewout Vansteenkiste and Wilfried Philips \\ Ghent University, Department of Telecommunications and Information Processing \\ TELIN-IPI-IBBT, Sint-Pietersnieuwstraat 41, Gent, Belgium
}

\begin{abstract}
In this paper, we propose a new algorithm for objective blur estimation using wavelet decomposition. The central idea of our method is to estimate blur as a function of the center of gravity of the average cone ratio (ACR) histogram. The key properties of ACR are twofold: it is powerful in estimating local edge regularity, and it is nearly insensitive to noise. We use these properties to estimate the blurriness of the image, irrespective of the level of noise. In particular, the center of gravity of the ACR histogram is a blur metric. The method is applicable both in case where the reference image is available and when there is no reference. The results demonstrate a consistent performance of the proposed metric for a wide class of natural images and in a wide range of out of focus blurriness. Moreover, the proposed method shows a remarkable insensitivity to noise compared to other wavelet domain methods.
\end{abstract}

Keywords: blur estimation, image quality, wavelets

\section{INTRODUCTION}

In the environment of rapidly growing demand for high quality and best performance service in the domain of digital image processing it is of great practical importance to develop algorithms for efficient assessment of image quality. One of the most common causes of image quality distortion is image blur which is inescapably introduced not only at the point of image acquisition but also during the iterations of image processing. Consequently, blur estimation is high on the list of priorities of image quality analysis.

Image degradation is usually observed twofold, as image noise and as image blur. If we denote a two-dimensional original image by $f(x, y)$ and a degraded one by $g(x, y)$, the degradation model can be described as

$$
g(x, y)=f(x, y) * h(x, y)+n
$$

where $*$ denotes the operation of two-dimensional linear convolution. Here $h(x, y)$ stands for the blurring function assumed to act as a convolution kernel or a point-spread function (PSF), and $n$ is the noise that corrupts the blurred image.

The types of blur which are commonly considered and regularly encountered in practical situations of interest are overviewed in [1]. These include linear motion blur, atmospheric turbulence blur often modeled by a Gaussian function and uniform out-of-focus blur. In this work, we will focus on out-of-focus blur as one of the most conventional blur that occurs in images. This type of blur is frequently introduced by a defocused camera and lens aberrations. It is often modeled as a circular averaging filter (pillbox) within the square matrix of side $2 r+1$, where $r$ is the radius of circular blur. In sense of the degradation model (1) the out-of-focus blur operator $h(x, y)$ may be defined as

$$
h(x, y)=\left\{\begin{array}{cc}
0, & \sqrt{x^{2}+y^{2}}>r \\
\frac{1}{\pi r^{2}}, & \text { otherwise }
\end{array} .\right.
$$

Clearly, the process of image restoration is aimed to reconstruct or estimate the ideal image $f(x, y)$ from a degraded one. More specifically, the objective of blur estimation methods is to identify attributes of the blur observed in a degraded image. It is important that these remain not influenced by the effects of noise which is commonly present in the degraded images. The combination of image restoration and blur identification is often referred to as blind image deconvolution.

Wavelet Applications in Industrial Processing VI, edited by Frederic Truchetet, Olivier Laligant, Proc. of SPIE-IS\&T Electronic Imaging, SPIE Vol. 7248, 72480F · @ 2009 SPIE-IS\&T · CCC code: 0277-786X/09/\$18 · doi: 10.1117/12.807412 
A large and growing body of literature has investigated blur estimation techniques. For example, one of the earlier studies [2] proposed using generalized cross validation to estimate the blur. Later, the method proposed by Panchapakesan et al. [3] used distortion of vector quantizer encoder to select the best PSF from a set of candidate ones. Li et al. [4] found that the kurtosis as a measure of how outlier-prone a distribution is may be used as an indication of blur in the image. Recently, blur identification based on spectral techniques was researched in [5].

Many of the existing methods strongly depend on the results of edge detection in the degraded image. For example, Rooms et al. [6] and later Tong et al. [7] use the ability of wavelet transform to detect location of the edges. Marziliano et al. [8], and more recently Wang et al. [9] make use of rather simple techniques of Canny or Sobel filtering to determine the edge positions. These edge detection techniques are affected by the level of noise in the degraded image. Hence, the related blur metrics are sensitive to noise as well.

Depending on the criterion they use for determining the level of blur, we recognize direct methods which look at certain discriminative features of the image and indirect ones which attempt to estimate blur by reconstructing the PSF. The latter ones include [2], [3], [5], [6], [10], [11]. The method proposed in this study is focused on estimating the level of blurriness in the image rather than estimating the PSF of the imaging system.

Multiple blur estimation methods require an iterative process where identification and restoration steps are performed sequentially [2], [3]. Recently, [11] proposed a method for iterative estimation of the blur which demonstrates high robustness to noise. Unlike these, we propose a metric which is based on a one time calculation of the metric value.

Eventually, we look at the use case scenario. In general, the metrics are classified into reference, reduced reference and no-reference ones. In contrast to the human observers, mathematical models are faced with a number of problems in the task of quality parameter evaluation of a distorted image when the original one is not available as a reference. A number of existing algorithms for image quality assessment belong to the class of reference based metrics, for example [8], [9]. As we will show later, the method proposed in this study falls in the class of both reference and no-reference ones.

The paper is organized as follows. In the next Section, we introduce the new blur metric and demonstrate its robustness to the image blur. In Section 3, we present the experiments conducted in the study and report the results. We then discuss the results in Section 4 and conclude with some final remarks in Section 5.

\section{THE PROPOSED BLUR METRIC}

In digital images, blur is usually observed as a loss of image sharpness which corresponds to smoothening of the edges. In mathematics, interpretation of an edge often refers to the singularities. Jaffard [12], Mallat, Hwang, and Zhong [13], [14] explained mathematical characterization of singularities with Lipschitz exponent. They showed that the maxima of the wavelet transform modulus can detect the location of the irregular structures and proposed a numerical procedure to estimate local Lipschitz exponents of these irregularities. This early approach was based on a computationally demanding procedure. Later on, several techniques have been devised to simplify and speed up the original algorithm. In the following, we make use of one such technique which exhibits significant robustness to noise.

\subsection{The ACR metric}

In particular, our proposed method makes use of a wavelet domain local regularity measure named average cone ratio (ACR). The ACR is defined by Pižurica et al. [15] as a quantity which characterizes joint expansion of the magnitudes of wavelet coefficients inside a cone of influence which is centered at the given spatial position. Let us denote by $\mathrm{C}(\mathrm{j}, \mathrm{l})$ the discrete set of wavelet coefficients at the resolution scale $2^{1}$, which belong to the cone of influence of the spatial position l. The magnitude of a wavelet coefficient is denoted by $\left|\mathrm{w}_{\mathrm{j}, \mathrm{m}}\right|$. For each spatial position $\mathrm{l}$, the ACR measure between any two dyadic scales $2^{\mathrm{n}}$ and $2^{\mathrm{k}}$, where $\mathrm{n}, \mathrm{k} \in \mathrm{Z}$ and $\mathrm{k} \geq \mathrm{n}+1$ is defined as

$$
\beta_{\mathrm{n} \rightarrow \mathrm{k}, \mathrm{l}} \triangleq \log _{2}\left(\frac{1}{\mathrm{k}-\mathrm{n}} \sum_{\mathrm{j}=\mathrm{n}}^{\mathrm{k}-1} \frac{\left|\mathrm{I}_{\mathrm{j}+1, \mathrm{l}}\right|}{\left|\mathrm{I}_{\mathrm{j}, \mathrm{l}}\right|}\right), \mathrm{I}_{\mathrm{j}, \mathrm{l}} \triangleq \sum_{\mathrm{m} \in \mathrm{C}(\mathrm{j}, \mathrm{l})}\left|\mathrm{w}_{\mathrm{j}, \mathrm{m}}\right|
$$

for each spatial position as a measure of evolution of the of wavelet coefficients across and inside a cone of influence, centered at a given spatial position. 


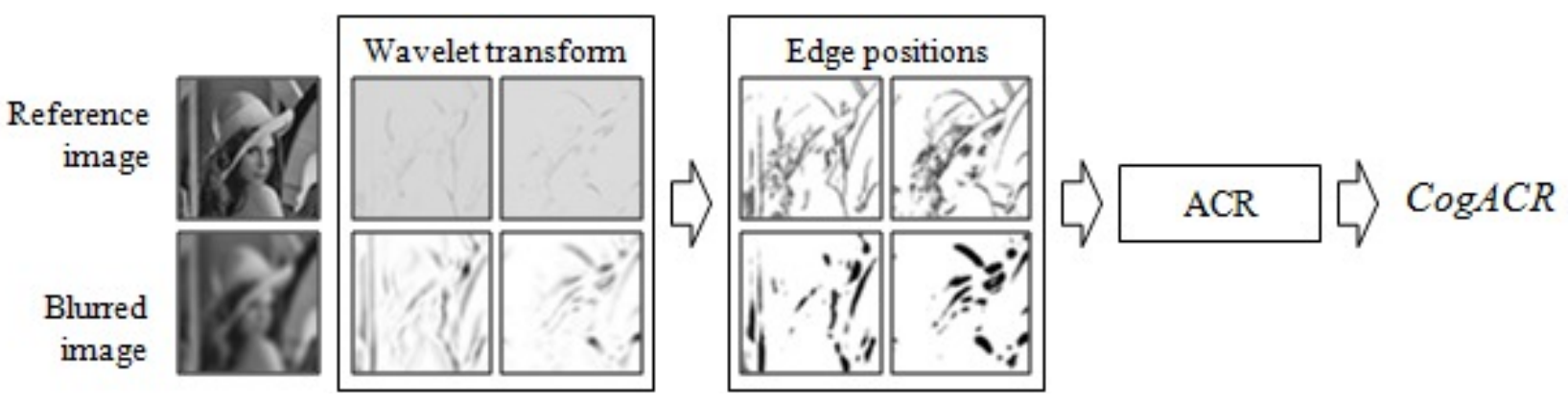

Fig. 1. Flowchart of the proposed algorithm for blur identification based on calculating the $\operatorname{Cog} A C R$ metric.

It was shown in [15] that the ACR is a good estimate of the local Lipschitz exponent $\alpha$, in particular an estimate of Lipschitz $\alpha+1$. Moreover, the study proved that the ACR measure is insensitive to noise. We make use of these properties of ACR (estimating local edge regularity while being insensitive to noise) for blur estimation. Fig. 2(c) illustrates the potentials of this approach in case of out-of-focus blur. In this figure, histograms of ACR are plotted for a test image degraded by different amounts of blur, for a noise-free case and for a heavy noise case. The histograms show nearly insensitivity to noise, while clearly shifting with the increase of blur. Similar trends exist for Gaussian blur and linear motion blur as well. Other related methods that were based on estimating the local regularity in the wavelet domain [6], [7] suffered from sensitivity to noise whereas the approach that we propose demonstrates a clear potential to overcome this problem.

\subsection{The proposed noise insensitive blur metric}

Let us introduce the algorithm of ACR based blur detection, see Fig. 1. First, we have to identify positions of the edges in the image of interest. As often is the case in edge detection tasks, we start from wavelet decomposition of an image to obtain two detail images at each scale, one with horizontal and one with vertical direction details. Note that, as shown in Fig. 1, this step may be applied on a reference non-degraded image or directly on the degraded image itself, depending on the availability. After thresholding the two detail images, the edge positions are identified. We will further discuss the threshold selection in the following subsection. Second, we use the edge positions to calculate the ACR histogram for the degraded image. Finally, we determine the center of gravity of the ACR histogram to obtain a numerical metric of the level of blur in the degraded image.

As mentioned earlier, the ACR measure $\beta_{n \rightarrow k, 1}$ can be calculated for any two dyadic scales $2^{\mathrm{n}}$ and $2^{\mathrm{k}}$. In order to select the best suited one for our purpose, we compared multiple scale ranges. We apply the criteria of highest sensitivity to blur and lowest sensitivity to noise in the widest range of image degradation both blur- and noise-wise. In general, higher inter-scale measures tend to be more robust to noise and still manage to retain the sensitivity to blur even at higher levels of degradation. Fig. 2 illustrates these conclusions on the example of ACR measure between scales $2^{2}$ and $2^{3}$ (ACR2-3) and ACR measure between scales $2^{2}$ and $2^{4}$ (ACR2-4). The value ranges presented in graphs (b) and (d) are summary results calculated on the noise-free set of test images used in the experiments. The results shown in graphs (a) and (c) are calculated for the Lena image to demonstrate noise sensitivity of the two ACR metrics associated with the level of blur in the image. We notice that ACR2-4 exhibits noticeably higher robustness to noise compared to ACR2-3. Additionally, the scope of values of ACR2-4 indicates superior sensitivity to blur in the range of higher blur levels while successfully discriminating the very low levels of blur as well. These considered we decide to use the ACR2-4 as a basis for our proposed metric. We call this metric $\operatorname{Cog} A C R$ hereafter.

\subsection{Extracting valid edges for ACR analysis}

As indicated in Fig. 1, before calculating the ACR histograms for the image we use wavelet decomposition of the image to determine the edge positions. Commonly, the positions of detected edges are represented by a binary mask. The mask $\mathbf{x}$ is a set $\left\{x_{1}, \ldots, x_{n}\right\}$ of binary labels: $x_{l}=1$ if $\left|w_{l}\right|$ is greater than the threshold $T$ and $x_{l}=0$ otherwise, where the set of indices $\{1, \ldots, n\}$ is a set of pixels on a regular rectangular lattice. 

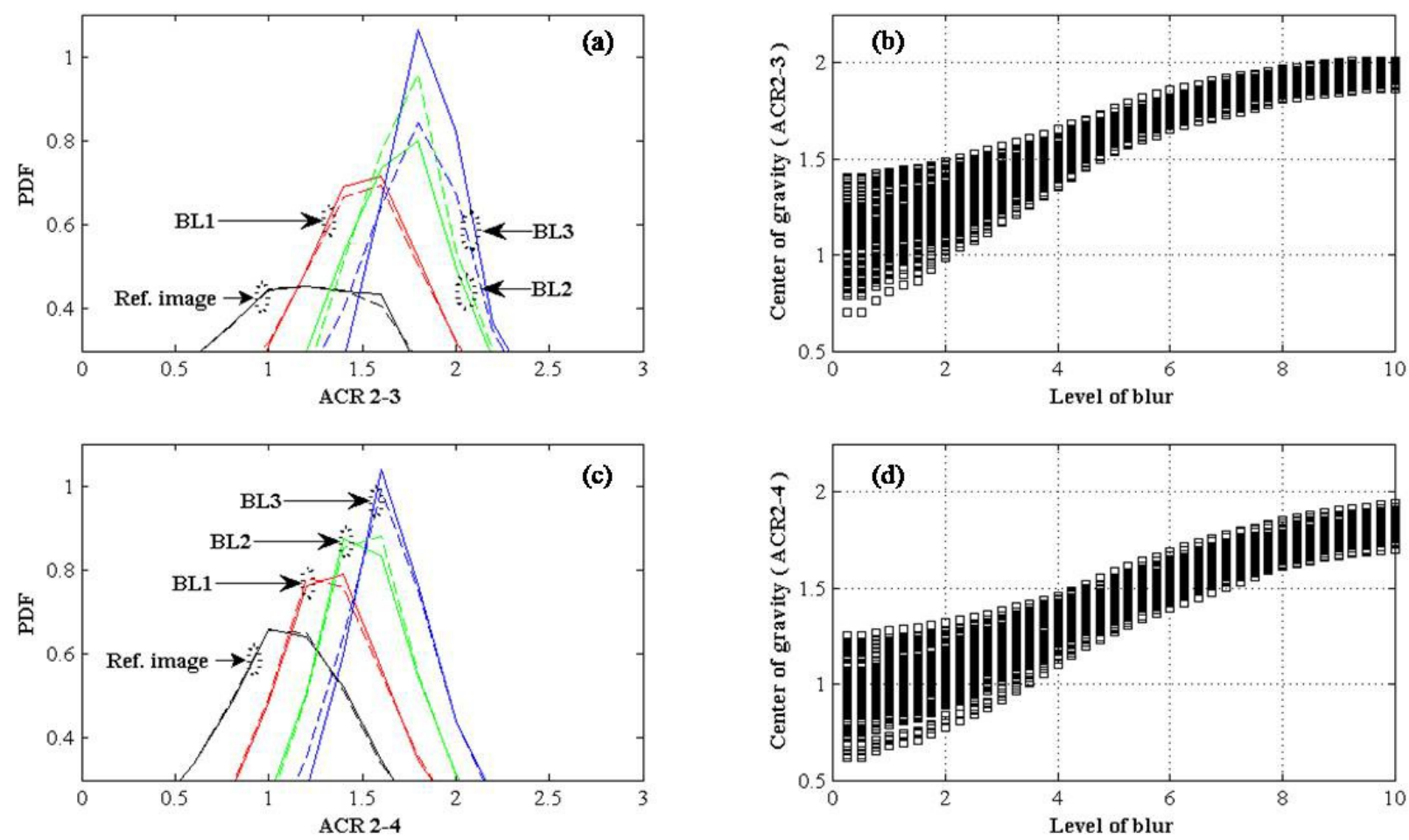

Fig. 2. Selecting the scales of ACR metric. (a) PDF of ACR2-3 measure different levels of blur in the image (BL1<BL2<BL3), (b) scope of values for ACR2-3, (c) PDF of ACR2-4 measure different levels of blur in the image $(\mathrm{BL} 1<\mathrm{BL} 2<\mathrm{BL} 3)$, (d) scope of values for ACR2-4. In (a) and (c) the solid lines stand for noise free image and the dashed lines stand for noisy images $\left(\sigma^{2}=10\right)$ both with different levels of blur (BL1<BL2<BL3). The three levels of blur BL1, BL2 and BL3 correspond to the circular blur with radius $r_{1}=3, r_{2}=5$ and $r_{3}=7$ respectively.

In the process of mask extraction, we distinguish between noise-free case and noise-present case. Assuming that the image we use in this step is noise-free, we could detect the edges by simply examining the detail images and identifying the positions of important wavelet coefficients. However, in case where the available image is noisy we need an alternative approach in order to allow for more reliable edge detection. In this case, the binary mask shall be estimated from an intermediate detail image acquired by multiplication of wavelet detail images at different scales, in particular the current scale and its adjacent one. Also, prior to scale multiplication, possible shifts introduced in the detail images by wavelet transform shall be compensated. In this study, we take the conservative approach and follow the procedure recommended for noisy images even in case of noise-free ones. In the following, the attribute "detail" is used with the wavelet coefficient to mean either the wavelet detail image or the wavelet scale multiplication, and the exact meaning should be determined by the presence of noise in the image of interest.

The next important consideration in the process of selecting the mask is image content, especially the type of edges in the image and frequency of the image texture. The mask should be selected to capture information about the major edges in the image rather than depicting those appearing very smooth compared to the dominant ones. Moreover, the mask should omit the details from very high frequency textures in the image.

For selecting the masks, we propose a simple content adaptive procedure that refers to the magnitude of wavelet coefficients. Namely, the threshold for extracting valid edges for ACR analysis shall be selected to preserve a certain percentage of detail wavelet coefficients with highest magnitudes which we call the threshold percent coefficient and denote by $T p$. In particular, our experimental study showed that the sufficient search space for percent coefficient is defined with $T p=\{0.01,0.05,0.10,0.15,0.20\}$. As the value of $T p$ increases, the absolute value of the threshold $T$ will decrease to allow more wavelet coefficients to be included in the mask. For example, in our study we use images of 256 x 256 pixels. In case where the threshold percent coefficient is selected to be $0.10\left(T p_{1}=0.10\right)$ the number of peak wavelet coefficients to be included in the mask is calculated as $(256 \times 256)$ x $0.10=6553.6$ which is rounded to 6554 . 
The wavelet coefficients of each detail image are than sorted in descending order according to their magnitude and the threshold is determined as the value of the coefficient positioned 655 in this list, $T_{1}=$ Const $_{1}$. If in the other case we would select the threshold percent coefficient to be $0.20\left(T p_{2}=0.20\right)$, the value of threshold would correspond to the value of detail wavelet coefficient ordered 13107 in the list, $T_{2}=$ Const $_{2}$. We conclude this numerical example with the observation that Const $_{2}>$ Const $_{1}$ which corresponds to $T_{2}>T_{1}$ and conforms to the statement of inverse relationship between the threshold $T$ and its percent coefficient $T p$.

\subsection{Degradation-free image available}

With respect to the usage scenario, the proposed blur metric is valid both in case where the reference image is available and in case where it is not available. We call this reference scenario (RS) and no-reference scenario (NRS), respectively.

In case where the degradation-free image is available, we use it as a reference to determine the positions of the relevant edges in the image, all as explained above. We then calculate the ACR histograms and their center of gravity to obtain the $\operatorname{Cog} A C R$ metric for the degraded image of interest. The value of $\operatorname{Cog} A C R$ will indicate the level of blur in the degraded image.

In view of the proposed metric, the natural scene images used in the study follow the trends shown in Fig. 2(d). Based on the range of sensitivity to lower levels of blur $(r \leq 4)$, we distinguish approximately two classes of images: a class of images with lower sensitivity to low level blurs, hereafter named class $A$, and a class of images with higher sensitivity to low level blurs, hereafter named class B. Class A refers to the upper part of the value space identified in Fig. 2(d), while class B denotes the remaining lower part of the value space.

\subsection{Degradation-free image not available}

When the original degradation-free image is not available, we follow a procedure similar to RS case except that now we use the degraded image to determine the edge positions. We briefly review the algorithm.

The starting point is the blurred image itself. First, we calculate wavelet transform to obtain the edge coefficients. Next, we determine the mask from these coefficients in the same manner we would do it when the non-blurred image is available. We remind that in case of noisy images the masks are obtained by thresholding the scale multiplication of the relevant wavelet coefficients, all as described in subsection 2.3. In the next step, we calculate the ACR of the degraded image using these masks. Finally, we estimate the level of blur by mapping the calculated $\operatorname{Cog} A C R$ value to its corresponding level of blur in the space of values (Fig. 2(d)). However, before we can conclude the process, it is necessary to determine the class of the degraded image. This refers to the classification between class A and class B, and is discussed in more details in the following sections.

\section{EXPERIMENTAL RESULTS}

The proposed metric, $\operatorname{Cog} A C R$, has been analyzed in the experimental study. The descriptions of the experiments and their goals as well as the summary results are presented in this section.

The test sample consisted of 130 natural gray scale images size 256 x 256 pixels. Fig. 3 illustrates some randomly selected examples from the test sample. The data set is comprised of the images taken from [16] and some author's owned images, both taken with digital cameras, together with some common images in the domain of image processing. As mentioned before, we focus on images with uniform out of focus blur modeled by (2). For this study, the value of blur radius is varied in the range of 0.25 pixels to 10.0 pixels with the uniform step of $0.25, r=\left\{r \in \mathbb{R}: r_{m}=0.25 m, m=\right.$ $1,2, \ldots, 20\}$.

\subsection{Edge extraction}

In Fig. 4 we show two examples of influence of the mask on $\operatorname{Cog} A C R$ metric. We notice different effects for image (a) compared to the image (b). In one case, the increase of threshold percent coefficient results in superior sensitivity to lower levels of blur and slightly degraded one as the level of blur grows (Cactus image). In another case, we notice that the sensitivity to blur drastically falls or even becomes slightly unstable with the increase of threshold percent coefficient (Girl image). To explain these differences, we analyze the content of the two images, especially in terms of their texture and the dominant type of edges in the image. 

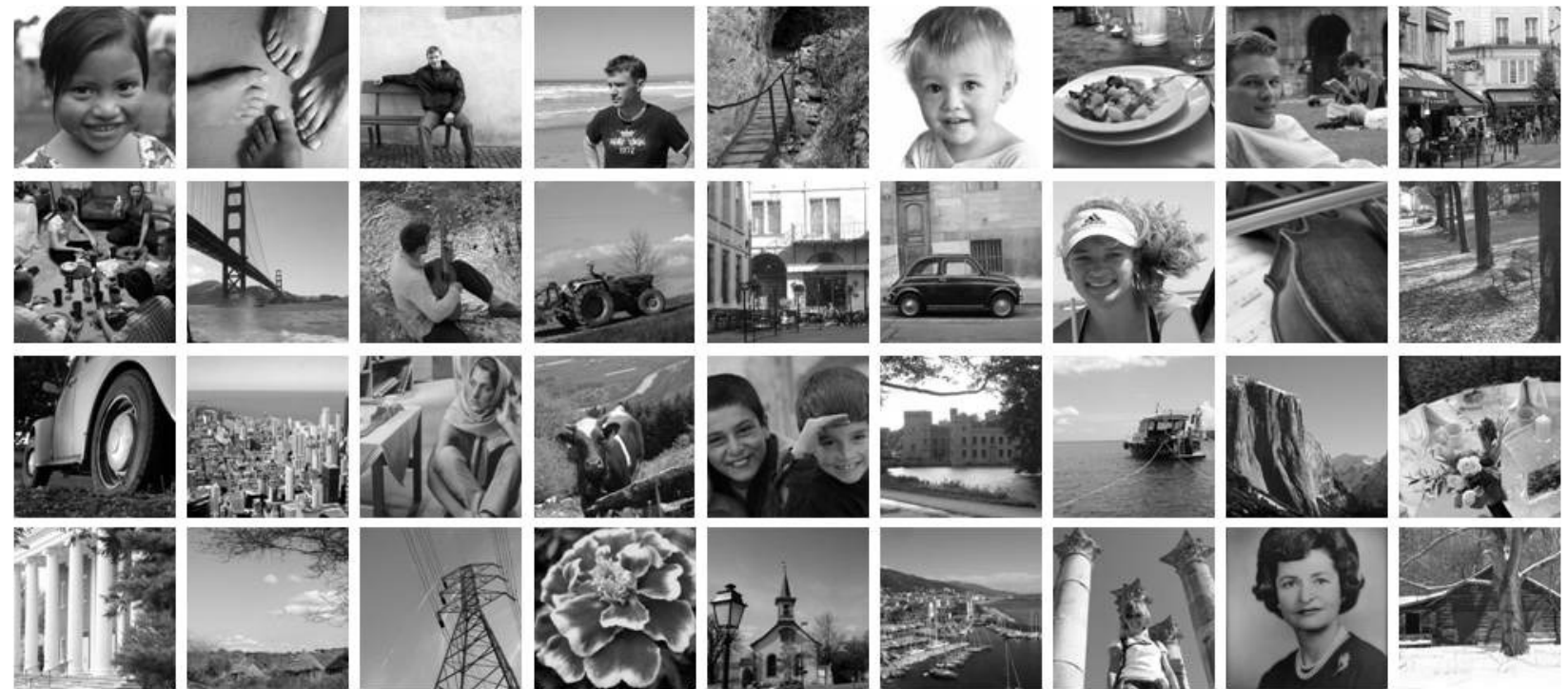

Fig. 3. Randomly selected 36 out of the total 130 gray scale images used in our experiments. All images are of size $256 \mathrm{x}$ 256 pixels.
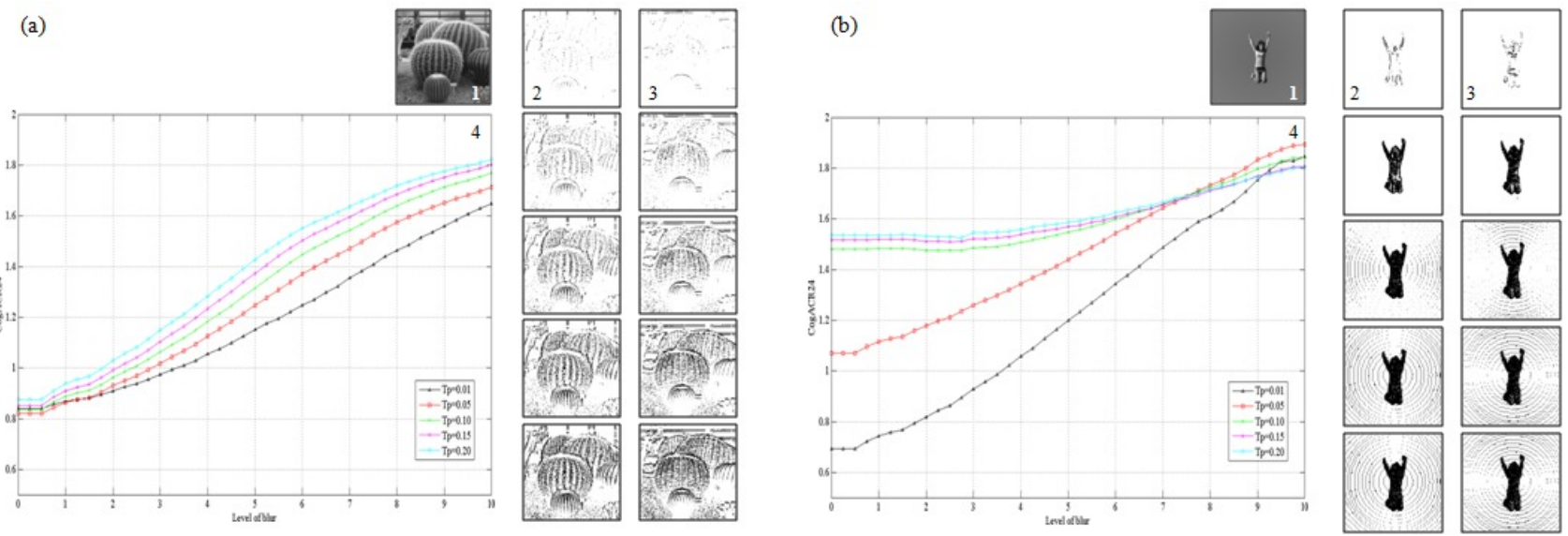

Fig. 4. $\operatorname{Cog} A C R$ metric conditional on mask selection. (a) Cactus image, (b) Girl image: 1 - Degradation free gray scale image; 2 - vertical edge positions detected from the non-degraded image for different values of threshold percent mask, $T p=1$ (top) to $T p=20$ (bottom), $T p=\{1,5,10,15,20\} ; 3$ - horizontal edge positions detected from the non-degraded image for values of $T p$ the same as in $2 ; 4-\operatorname{Cog} A C R$ metric for circular blur, $r=\{0,0.25,0.50, \ldots, 10\}$.

Compared to the Cactus image, we observe that the Girl one contains far less significant edges and mainly low frequency texture. This suggests that a higher percent of edges shall be considered for adequate identification of blur in the Cactus image. Looking at the response of $\operatorname{Cog} A C R$ to blur, for the Cactus image we observe the most stable and approximately linear dependency for $T p=0.10$ while for the Girl image this applies for $T p=0.05$. This aligns well with our conclusions based on the image contents. Finally, we look at the masks. We may easily notice that the corresponding masks capture sufficient information to allow for unambiguous visual matching between the original image and the extracted edges.

Experimental results of our study prove that the above explained analysis of the image content is a valid argument for selecting the optimal value of threshold percent coefficient for $\operatorname{Cog} A C R$. This confirms that image content analysis is a necessary precondition for adequate selection of the threshold percent coefficient value.

In our study, for 120 out of 130 images the value of $T p=0.10$ proved to be an optimal choice. 

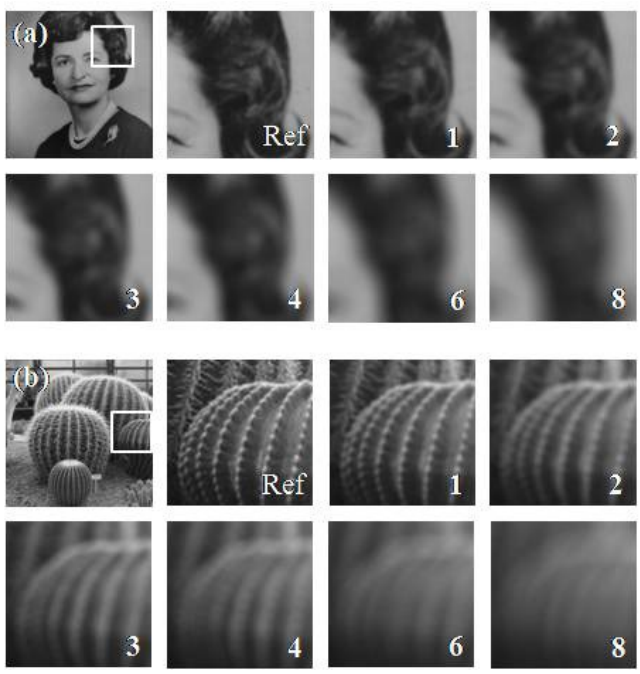

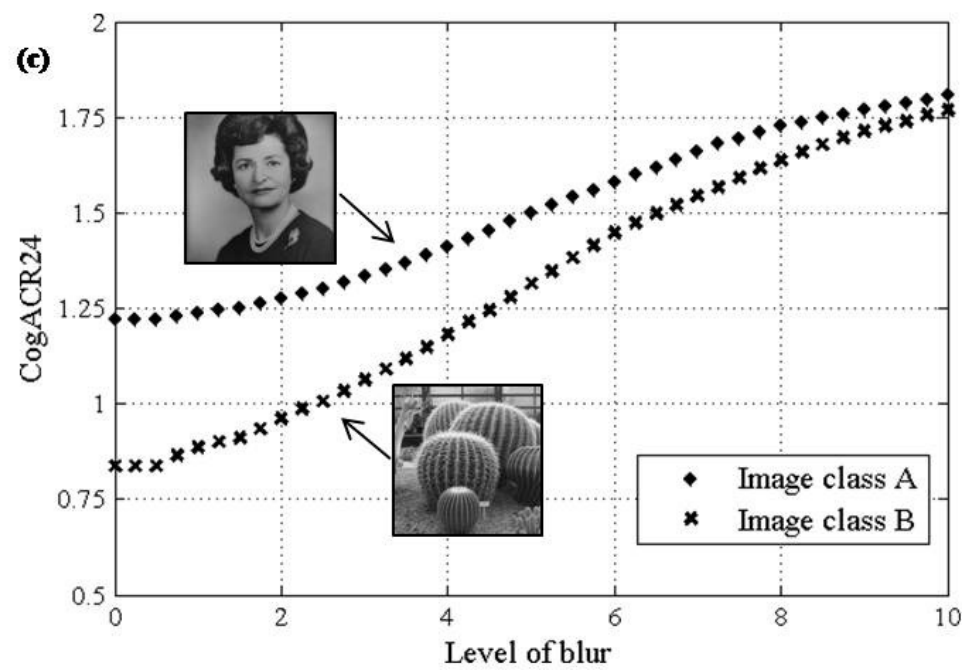

Fig. 5. Classification of images based on the $\operatorname{Cog} A C R$ sensitivity to lower levels of blurriness. (a) Example image from class A (Face image), (b) example image from class B (Cactus image), the numbers in upper lower corner of the images indicate the value of circular blur radius for a given image segment. (c) $\operatorname{Cog} A C R$ curves for image (a) and image (b).

\section{2 $\operatorname{CogACR}$ in the reference scenario}

The goal of this experiment is to determine the dependency of the proposed blur metric to the image content, especially on the texture of image content. Example images for each of the two classes, class A and class B, are shown in Fig. 5(c). In addition, we show the enlarged segment of each image for increasing values of the radius of the blur, $r=\{1,2,3,4,6,8\}$ (Fig. 5 (a) and (b)). These segments are aimed to give evidence for different effect of blur in relation with the strength of the edges and frequency of the texture in the images. We observe that the same quanta of increase of the blurriness is more clearly perceived in higher frequency content where the mask necessarily consists of a great number of medium to soft edges (Cactus image) than in the lower frequency one where the mask is allowed to depict fewer edges by selecting only the hard ones (Face image). Remember, the mask shall reliably portray the content of the image so that the $\operatorname{Cog} A C R$ can confidently estimate the level of degradation in the image. Inevitably, this translates to a greater amount of less strong edges in the mask for the high frequency texture, and vice versa, fewer strong edges in the mask for the texture of low frequency.

Having identified this dependency, we conclude that the frequency of image texture may be used as a relevant feature for the selection of image class in view of $\operatorname{Cog} A C R$ metric, especially in case of NRS.

\section{3 $\operatorname{Cog} A C R$ in the no-reference scenario}

This group of experiments investigates performance of the $\operatorname{Cog} A C R$ metric in case where the degradation free image is not available. Furthermore, we analyze the influence of noise in such scenario.

Fig. 6 compares the $\operatorname{Cog} A C R$ values obtained in case where the reference degradation-free image is available to those calculated from the degraded image. Again, we select one example image from class A and one from class B. As expected, in RS scenario there is slight deviation from the ideal case where the edges would be detected from the nondegraded image. Not surprisingly, this deviation is greatest in case o higher levels of blur. This is due to the fact that in the absence of blur free image the edge positions need to be defined from the degraded image itself. Inevitably, more significant blurriness in the image will affect the quality of edge detection and thus also influence the accuracy of the final $\operatorname{Cog}$ ACR measurement. Despite the observed deviations, the metric has preserved its ability to clearly discriminate between different considered levels of blur. Moreover, in this case the $\operatorname{Cog} A C R$ metric has not only retained but even increased its sensitivity to the degradation caused by very low levels of blur. 

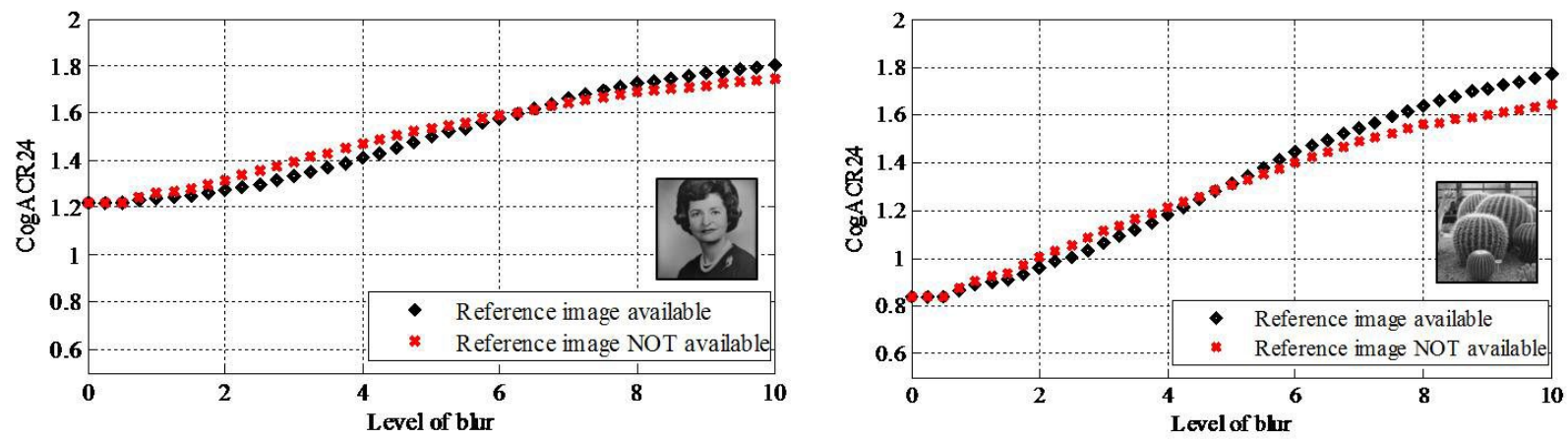

Fig. 6. $\operatorname{Cog} A C R$ metric in case of reference image not available. Comparison to the ideal degradation-free scenario is given.
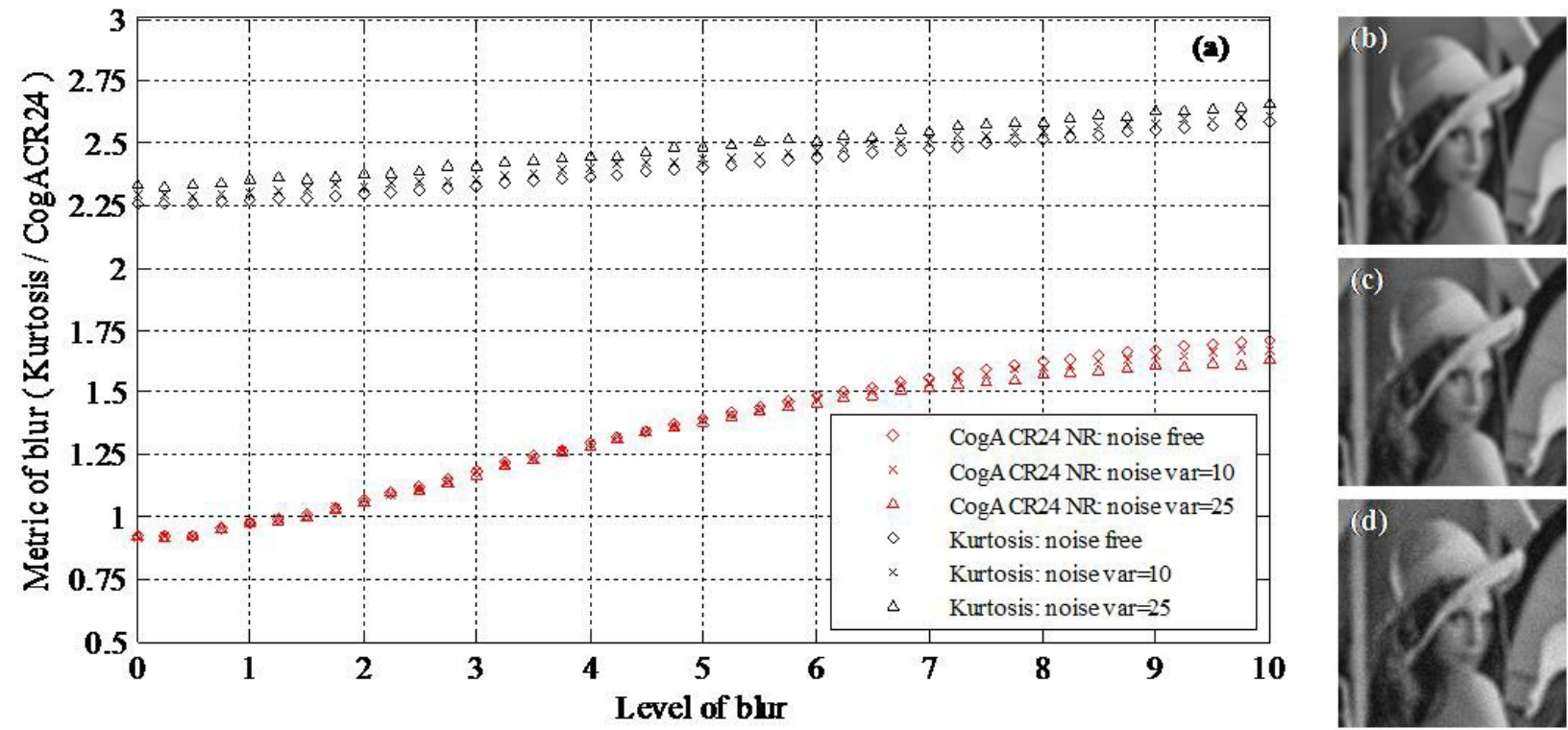

Fig. 7. $\operatorname{Cog} A C R$ metric compared to the kurtosis metric of blur. (a) $\operatorname{Cog} A C R$ metric calculated for NRS compared to the kurtosis metric, both in cases of: no noise degradation, Gaussian noise degradation with noise variance of 10 and Gaussian noise degradation with noise variance of 25. (b) Test image with no noise and level of circular blur $r=5$, (c) test image with Gaussian noise $\left(\sigma^{2}=10\right)$ and level of blur $r=5$, (d) test image with Gaussian noise $\left(\sigma^{2}=25\right)$ and level of circular blur $r=5$.

In Fig. 7 we compare the $\operatorname{Cog} A C R$ to the method of Li et al. [3]. Their technique is based on minimization of the kurtosis as a measure of blur. The results show that the $\operatorname{Cog} A C R$ calculated for NRS is able to distinguish between different levels of blur with higher level of confidence than the kurtosis metric while preserving very low noise sensitivity, especially in case of lower levels of blur. We may clearly observe that the $\operatorname{Cog} A C R$ metric is nearly insensitive to noise up to the level of blur characterized by the value of $r=6$ which in many real life cases is considered a high level of blur. This confirms the initial hypothesis of the study about high robustness to noise which stems from the characteristics of the ACR metric itself.

\section{DISCUSSION}

In the current study, a new metric for blur identification is proposed. A series of experiments was conducted to investigate out of focus blur discrimination in a range of image contents capturing natural scenes.

Key characteristics of the $\operatorname{Cog} A C R$ metric involve high level of discrimination between different levels of blur in the image for a significant range of blurriness, low sensitivity to noise, and ability to be used as both reference and no- 
reference metric while preserving the high sensitivity to blur and low sensitivity to noise. Other major points of interest investigated in the study together with the related findings are already discussed in the previous section while the results were presented.

In the study, we investigated the ability of the $\operatorname{Cog} A C R$ metric to discriminate between different levels of blur in a clear and unambiguous manner. This puts forward one possible application of the metric in the task of optimization and evaluation of different imaging systems with respect to the image quality deterioration they introduce in terms of blurring. Such application would involve a reference image and use the $\operatorname{Cog} A C R$ to measure the level of degradation introduced by the system. Another case of interest is a no-reference one where the $\operatorname{Cog} A C R$ may be used to estimate the level of blurring in the degraded image so that the estimated quantity of blur is then available as an input for algorithms which, unlike blind deconvolution for example, require prior information about the degraded image.

At this stage of the development, we use the visual arbitration of image content, more specifically image texture, to decide about the class of the image in case where the degradation-free image is not available. Further investigation in this direction is ongoing. Beside from image texture, we are looking not only at the histograms of the available gray level image but also at the features of ACR histogram of the decomposed image.

To end with, we refer to the description of the data set used in this study given in section 3. It says that most of the images are real rather than the ideal ones which imply that some blur may be present already in the reference data. We may want to avoid this in future experiments and consider using ideal images only for further accuracy of the results.

\section{CONCLUSIONS}

The present study was designed to determine the performance of a newly proposed blur metric named $\operatorname{CogACR}$ in the scenario of both reference image available and no-reference image available. In particular, the potential of high robustness to noise was to be verified.

One of the more significant conclusions drawn from the results of the study is that the $\operatorname{Cog} A C R$ may be used as a powerful metric of image blur over a wide range of blur levels and while being nearly insensitive to noise. Moreover, the results of the study prove the ability of the new blur metric to clearly discriminate between low level quanta of blur in both reference- and no-reference case which encourages further research of the subject.

In particular, further studies need to be done to investigate methods for classifying the images in case where the degradation free image is not available. In addition, possible techniques for automated selection of the threshold percent coefficient shall be addressed. Finally, we mention that the proposed technique has been successfully used in some non natural scene images as well. Further investigation of these types of image content is subject to a further study.

\section{REFERENCES}

[1] Lagendijk, R. L. and Biemond, J., "Basic methods for image restoration and identification," in Handbook of Image and Video Processing, J. D. Gibson, A. Bovik, Academic Press, Inc. Orlando, FL, USA (2000).

[2] Reeves, S. J. and Mersereau, R. M., "Blur identification by the method of generalized cross-validation," IEEE Trans. Image Processing vol.1, 301-311 (1992).

[3] Panchapakesan, K., Marcellin, M. W. and Hunt, B. R., "Blur identification from vector quantizer encoder distortion," IEEE Trans. Image Processing vol.10, 465-470 (2001).

[4] Li, D., Mersereau, R. M. and Simske, S., "Blur identification based on kurtosis minimization," Proc. IEEE ICIP I, 905-8 (2005).

[5] Van Zyl Marais, I. and Steyn, W. H., "Robust defocus blur identification in the context of blind image quality assessment," Image Commun. vol. 22, 833-844 (2007).

[6] Rooms, F., Pižurica, A., and Philips, W., "Estimating image blur in the wavelet domain," Proc. of the Fifth Asian Conference on Computer Vision (ACCV), pp. 210-215 (2002).

[7] Tong, H., Li, M., Zhang, H., and Zhang, C., "Blur detection for digital images using wavelet transform," Proc. ICME, 17-20 (2004). 
[8] Marziliano, P., Dufaux, F., Winkler, S., Ebrahimi, T. and Sa, G., "A no-reference perceptual blur metric," Proc. ICIP, 57-60 (2002).

[9] Wang, X., Tian, B., Liang, C. and Shi, D., "Blind Image Quality Assessment for Measuring Image Blur," Proc. of the 2008 Congress on Image and Signal Processing (CISP) vol. 1, 467-470 (2008).

[10] Devcic, Z. and Loncaric, S., "Blur identification using averaged spectra of degraded image singular vectors," Proc. ICASSP, 2195-2198 (2000).

[11] Moghaddam, M., "A robust noise independent method to estimate out of focus blur," Proc. ICASSP (2008).

[12] Jaffard, S., "Pointwise smoothness, two-microlocalization and wavelet coefficients," Publicacions Matemàtiques vol. 35, 155-168 (1991).

[13] Mallat, S., and Zhong, S., "Characterization of Signals from Multiscale Edges," IEEE Trans. Pattern Anal. and Machine Intel. vol. 14, 710-732 (1992)

[14] Mallat, S. and Hwang, W. L., "Singularity detection and processing with wavelets," IEEE Trans. Information Theory vol. 38, 617-643 (1992).

[15] Pižurica, A., Philips, W., Lemahieu, I. and Acheroy, M., "A Joint Inter- and Intrascale Statistical Model for Bayesian Wavelet Based Image Denoising," IEEE Trans. on Image Processing vol. 11, no. 5, 545-557 (2002).

[16]D. Niblack, http://imagebase.davidniblack.com 